\title{
Activité anti-inflammatoire de l'extrait aqueux des feuilles de Elaeis guineensis Jacq. (ARECACEAE) sur l'œdème aigu de la patte de rat induit par la carraghènine
}

\author{
Madièye SENE ${ }^{1 *}$, Mamadou NDIAYE ${ }^{1}$, Firmin Sylva BARBOZA ${ }^{1}$, Mbaye SENE $^{2}$, \\ William DIATTA ${ }^{3}$, Abdou SARR ${ }^{3}$, Awa NDIAYE-SY ${ }^{1}$, Amadou Moctar DIEYE $^{1}$ et \\ Guata Yoro SY ${ }^{1}$ \\ ${ }^{1}$ Laboratoire de Pharmacologie et Pharmacodynamie, Faculté de Médecine, de Pharmacie et d'Odontologie, \\ Université Cheikh Anta Diop, BP 5005, Dakar, Sénégal. \\ ${ }^{2}$ Laboratoire de physiologie, Faculté de Médecine, de Pharmacie et d'Odontologie, Université Cheikh Anta \\ Diop, BP 5005, Dakar, Sénégal. \\ ${ }^{3}$ Laboratoire de Pharmacognosie et Botanique, Faculté de Médecine, de Pharmacie et d'Odontologie, \\ Université Cheikh Anta Diop, BP 5005, Dakar, Sénégal. \\ *Auteur correspondant ; E-mail : s.madieye@gmail.com
}

\section{RESUME}

Elaeis guineensis est souvent utilisée en médecine traditionnelle africaine dans la prise en charge de nombreuses pathologies. Ces travaux avaient pour objectif de faire une étude phytochimique et de déterminer l'activité anti-inflammatoire de l'extrait aqueux des feuilles de Elaeis guineensis. Le modèle de l'œdème inflammatoire de la patte de rat induite par la carraghénine a été utilisé pour cette étude. L'extrait aqueux des feuilles de Elaeis guineensis Jacq. a été utilisé aux doses de 300 et $500 \mathrm{mg} / \mathrm{kg}$. Après administration orale, cet extrait à la dose de $300 \mathrm{mg} / \mathrm{kg}$, prévient de manière significative $(\mathrm{p}<0,05)$ l'œè̀me de la patte de rat induite par la carraghénine. L'augmentation du pourcentage de l'œdème inflammatoire de la patte est de 25,94 $\pm 6,17$; $34,81 \pm 10,55$ et $51,65 \pm 16,93$ respectivement aux temps $T_{1 h}, T_{3 h}$ et $T_{6 h}$ après injection de la carraghénine. Elle est de $19,73 \pm 5,09 ; 24,32 \pm 4,20$ et $32,15 \pm 4,08$ à la dose de $500 \mathrm{mg} / \mathrm{kg}$ respectivement à $\mathrm{T}_{1 \mathrm{~h}}, \mathrm{~T}_{3 \mathrm{~h}}$ et $\mathrm{T}_{6 \mathrm{~h}}$ après injection de la carraghénine. Ces résultats montrent que les feuilles de Elaeis guineensis possèdent une activité anti-inflammatoire qui justifierait son utilisation en médecine traditionnelle africaine pour prévenir ou traiter l'inflammation.

(C) 2016 International Formulae Group. All rights reserved.

Mots clés : Elaeis guineensis, inflammation, carraghénine.

\section{Anti-inflammatory activity of aqueous extract of leaves of Elaeis guineensis Jacq. (ARECACEAE) in the carrageenan-induced rat paw edema}

\section{ABSTRACT}

Elaeis guineensis is often used in african traditional medicine in the treatment of many diseases. This work aimed to make a phytochemical study and determine the anti-inflammatory activity of aqueous extract of leaves of this plant. Carrageenan-induced rat paw edema model was used for this study. We tested the aqueous 
extract of the leaves at doses of 300 and $500 \mathrm{mg} / \mathrm{kg}$. Oral administration of aqueous extract of leaves at dose of $300 \mathrm{mg} / \mathrm{kg}$, prevents significantly $(\mathrm{p}<0.05)$ carrageenan-induced rat paw edema. Increasing percentage of inflammatory paw edema is $25,94 \pm 6,17 ; 34,81 \pm 10,55$ et $51,65 \pm 16,93$ respectively at $T_{1 h}, T_{3 h}$ et $T_{6 h}$ after carrageenan injection. It is $19,73 \pm 5,09 ; 24,32 \pm 4,20$ et $32,15 \pm 4,08$ at the dose of $500 \mathrm{mg} / \mathrm{kg}$ respectively at $\mathrm{T}_{1 \mathrm{~h}}$, $\mathrm{T}_{3 \mathrm{~h}}$ et $\mathrm{T}_{6 \mathrm{~h}}$ after carrageenan injection. These results show that Elaeis guineensis leaves have anti-inflammatory activity that justifies its use in african traditional medicine to prevent or treat inflammation.

(C) 2016 International Formulae Group. All rights reserved.

Keywords: Elaeis guineensis, inflammation, carrageenan.

\section{INTRODUCTION}

L'inflammation constitue une manifestation de multiples maladies. Les syndromes inflammatoires sont très fréquemment rencontrés en pratique courante. La réaction inflammatoire peut être associée à une très grande variété de situations pathologiques: infections, maladies de système, cancers, pathologies thromboemboliques (Coussens et Werb, 2002; Dandona et al., 2004 ; Karin et al., 2006).

Ainsi, bien qu'elle soit indispensable à la survie de l'organisme agressé, l'inflammation n'en est pas moins dangereuse (Galanaud, 2003). Les traitements utilisés, anti-inflammatoires non stéroïdiens (AINS) et anti-inflammatoires stéroïdiens (AIS) constituent des risques mais demeurent les médicaments les plus vendus (Renfrey et al., 2003).

En Afrique, Elaeis guineensis Jacq. est utilisée en médecine traditionnelle africaine dans la prise en charge de courbatures et dans les douleurs rhumatismales (Pousset, 2004). D'autres auteurs ont également mis en évidence les activités antioxydante et antibactérienne (Yin et al., 2013), antidiabétique (Rosalina et al., 2011), antihypertensive (Ndiaye et al., 2010), anticancéreuse et anti-inflammatoire (Gruca et al., 2014 ; Owoyele et Owolabi, 2014) de Elaeis guineensis Jacq.

L'objectif de cet étude était de réaliser une étude phytochimique et d'évaluer l'activité anti-inflammatoire de l'extrait aqueux des feuilles de Elaeis guineensis sur un modèle animal d'œdème aigu de la patte de rat induit par la carraghénine.

\section{MATERIELS ET METHODES Matériel végétal}

Il est constitué par les feuilles de Elaeis guineensis. Elles ont été récoltées au niveau du Jardin Botanique de la Faculté de Médecine, de Pharmacie et d'Odontologie de l'Université Cheikh Anta DIOP de Dakar. L'identification botanique a été faite au laboratoire de Pharmacognosie et de Botanique de la dite faculté.

Les feuilles ont été séchées dans le laboratoire à la température ambiante $\left(25^{\circ} \mathrm{C}\right.$ environ) pendant 3 semaines. Les feuilles séchées ont ensuite été pulvérisées, donnant une poudre avec laquelle l'extrait aqueux a été préparé.

\section{Préparation des extraits}

La poudre des feuilles de Elaeis guineensis $(100 \mathrm{~g})$ a été mélangée avec un (01) litre d'eau distillée et porté à ébullition pendant 30 minutes puis filtré après refroidissement. Le filtrat obtenu a été concentré à l'aide d'un rotavapor (température fixée à $90{ }^{\circ} \mathrm{C}$ ). L'extrait pâteux obtenu a ensuite été séché au dessiccateur pour obtenir un extrait sec. Ce dernier, après réduction, a donné une poudre d'extrait aqueux sec (EAEG). Le rendement obtenu après extraction a été de $14,78 \%$.

\section{Animaux}

Des rats adultes de souches Wistar dont le poids est compris entre 110 et $190 \mathrm{~g}$ ont été utilisés pour cette étude. Ces rats ont été élevés à l'animalerie du Laboratoire de Pharmacologie et Pharmacodynamie avec un libre accès à la nourriture et à l'eau. 


\section{Protocole expérimental d'étude de l'activité anti-inflammatoire}

L'activité anti-inflammatoire a été évaluée in vivo, par la méthode de l'œdème de la patte de rat induit par la carraghénine (Winter et al., 1962). Les rats répartis en 4 lots de 5 , ont été pesés puis mis à jeun 12 heures avant l'expérimentation.

Pour chaque rat, le volume initial $\left(\mathrm{V}_{0}\right)$ de la patte postérieure gauche a été mesuré à l'aide d'un Pléthysmomètre à eau (APELEX 05-7150, Allinde, Bagneux, France) avant l'administration des traitements. Les différents traitements ont été administrés par gavage :

- Lot 1: eau physiologique en raison de $10 \mathrm{~mL} / \mathrm{kg}$ (contrôle)

- Lot 2: solution d'extrait aqueux de Elaeis guineensis à la dose de $300 \mathrm{mg} / \mathrm{kg}$

- $\quad$ Lot 3: solution d'extrait aqueux de Elaeis guineensis à la dose de $500 \mathrm{mg} / \mathrm{kg}$

- Lot 4: solution d'acide acétylsalicylique à la dose de $150 \mathrm{mg} / \mathrm{kg}$.

Trente minutes après l'administration orale des différentes solutions, chaque rat a reçu une injection de $100 \mu \mathrm{l}$ de solution de carraghénine à $1 \%$ sous le coussinet plantaire de la patte postérieure gauche.

Les volumes des pattes injectées ont été mesurés 60,180 et 360 minutes $\left(\mathrm{T}_{1 \mathrm{~h}}, \mathrm{~T}_{3 \mathrm{~h}}\right.$ et $\mathrm{T}_{6 \mathrm{~h}}$ ) après injection de carraghénine, toujours à l'aide d'un Pléthysmomètre à eau (APELEX 05-7150, Allinde, Bagneux, France).

L'importance de l'œdème a été appréciée par la détermination de la moyenne du pourcentage d'augmentation (\%AUG) du volume de la patte de rat suivant la formule :

$\%$ AUG $=\frac{V t-V_{0}}{V_{0}} \times 100$

Vt : Volume de la patte au temps $\mathrm{t}$; $\mathrm{V}_{0}$ : Volume initial de la patte

L'activité anti-inflammatoire a été évaluée par le calcul du pourcentage d'inhibition (\%INH) de l'œdème selon la formule :

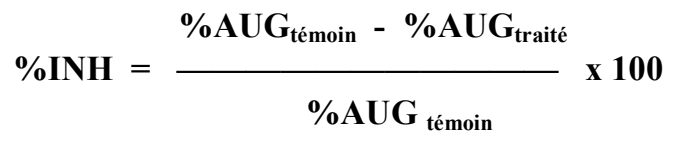

\section{Screening phytochimique}

La chlorure ferrique $\left(\mathrm{FeCl}_{3}\right)$ est utilisée pour la mise en évidence des tanins, la réaction à la cyanidine (alcool chlorhydrique) pour la caractérisation des flavonoïdes et la réaction de Dragendorff pour la mise en évidence des alcaloïdes.

\section{Analyse statistique}

Les résultats ont été exprimés en moyenne \pm erreur standard à la moyenne (e.s.m.). Les différences entre les deux moyennes ont été déterminées par analyse de variance ANOVA, suivi d'un test de comparaison multiple de Bonferroni pour les groupes à l'aide du logiciel GraphPad Prism5. Une différence significative est représentée par un $\mathrm{p}<0,05$.

\section{RESULTATS}

\section{Screening phyto-chimique}

L'analyse chimique de l'extrait aqueux des feuilles de Elaeis guineensis a montré la présence de tanins (tanins hydrolysables ou tanins pyrogalliques et les tanins condensés non hydrolysables ou tanins catéchiques), d'hétérosides flavonoïdes et d'alcaloïdes.

\section{Effet de l'extrait aqueux des feuilles de Elaeis guineensis sur l'œè̀me inflammatoire de la patte de rat induite par la carraghénine}

L'administration de l'extrait aqueux des feuilles de Elaeis guineensis à la dose de 300 $\mathrm{mg} / \mathrm{kg}$ prévient de manière significative $(\mathrm{p}<0,05)$ l'œdème de la patte de rat induite par la carraghénine. L'augmentation du pourcentage de l'œdème inflammatoire de la patte est de $25,94 \pm 6,17 ; 34,81 \pm 10,55$ et $51,65 \pm 16,93$ comparée au groupe contrôle traité avec l'eau physiologique dont l'augmentation de l'œdème est de $31,28 \pm 4,53$; $73,74 \pm 4,91$ et $100,24 \pm 10,39$ respectivement 
aux temps $T_{1 h}, T_{3 h}$ et $T_{6 h}$ après injection de la carraghénine.

A la dose de $500 \mathrm{mg} / \mathrm{kg}$ per os, l'extrait aqueux de Elaeis guineensis montre une meilleure prévention de l'œdème de la patte induite par la carraghénine comparée à la dose de $300 \mathrm{mg} / \mathrm{kg}$. Les pourcentages d'augmentation de l'œdème inflammatoire de la patte sont moins importants, ils sont de : $19,73 \pm 5,09 \quad ; \quad 24,32 \pm 4,20 \quad$ et $32,15 \pm 4,08$ respectivement aux temps $T_{1 h}, T_{3 h}$ et $T_{6 h}$ après injection de la carraghénine (Tableau 1).
L'évaluation $\mathrm{du}$ pourcentage d'inhibition montre que l'extrait aqueux des feuilles de Elaeis guineensis possède une activité anti-inflammatoire plus importante dans la seconde phase $\mathrm{du}$ processus inflammatoire. Cependant une meilleure inhibition de l'œdème inflammatoire de la patte de rat a été observé à $6 \mathrm{H}$ (Figure 1) avec un pourcentage d'inhibition de $67,17 \pm 3,31$ comparée au groupe de référence traité avec l'acide acétylsalicylique $150 \mathrm{mg} / \mathrm{kg}$ qui montre une inhibition de 56,72 $\pm 10,57$.

Tableau 1 : Effet de l'extrait aqueux des feuilles de Elaeis guineensis Jacq. sur l'œdème de la patte de rat induit par la carragénine.

\begin{tabular}{ccccc}
\hline \multirow{2}{*}{ Lots } & \multirow{2}{*}{$\begin{array}{c}\text { Doses } \\
(\mathbf{m g} / \mathbf{k g})\end{array}$} & \multicolumn{3}{c}{$\begin{array}{c}\text { Pourcentage d'augmentation du volume } \\
\text { de la patte induite par la carragénine }\end{array}$} \\
\cline { 3 - 5 } & & $\mathbf{1 H}$ & $\mathbf{3 H}$ & $\mathbf{6 H}$ \\
\hline $\begin{array}{c}\text { Contrôle } \\
\text { Eau physiologique }\end{array}$ & $\mathbf{( 1 0} \mathbf{~ m L} / \mathbf{k g})$ & $31,28 \pm 4,53$ & $73,74 \pm 4,91$ & $100,24 \pm 10,39$ \\
\hline EAEG & $\mathbf{3 0 0}$ & $25,94 \pm 6,17$ & $34,81 \pm 10,55^{*}$ & $51,65 \pm 16,93$ \\
\hline EAEG & $\mathbf{5 0 0}$ & $19,73 \pm 5,09$ & $24,32 \pm 4,20^{*}$ & $32,15 \pm 4,08^{*}$ \\
\hline Aspirine & $\mathbf{1 5 0}$ & $9,12 \pm 10,51$ & $23,08 \pm 10,51^{*}$ & $42,86 \pm 11,74^{*}$ \\
\hline
\end{tabular}

Les données sont exprimés en moyenne \pm erreur standard à la moyenne (e.s.m.). Significativité à $* \mathrm{P}<0,05$ versus contrôle. $\mathrm{n}=5$

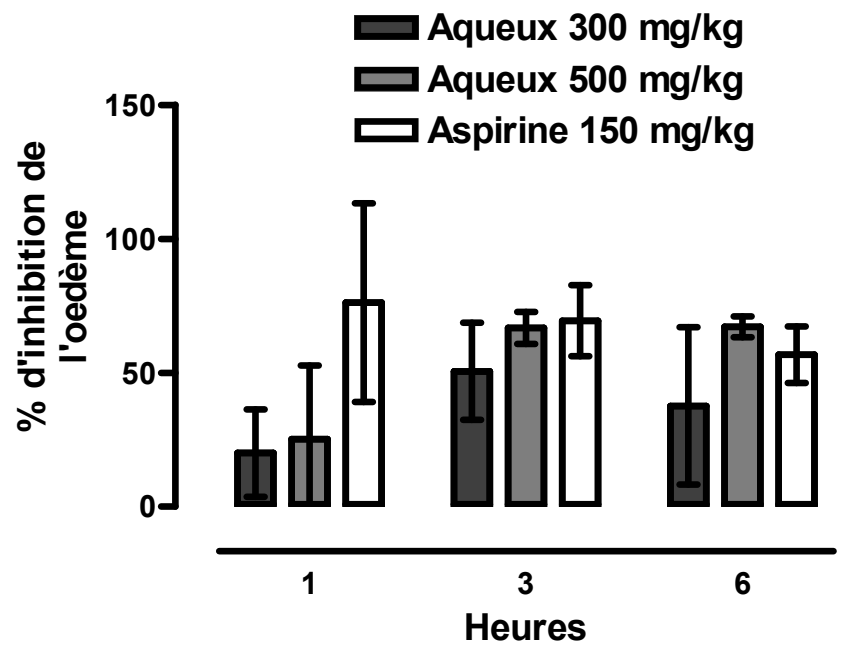

Figure 1 : Pourcentage moyen d'inhibition de l'œdème après administration d'extraits aqueux des feuilles de Elaeis Guineensis Jacq. aux doses de 300 et $500 \mathrm{mg} / \mathrm{kg}$ et de l'aspirine à la dose de 150 $\mathrm{mg} / \mathrm{kg}$ aux rats Wistar. 


\section{DISCUSSION}

L'administration per os de l'extrait aqueux des feuilles de Elaeis guineensis s'est révélée efficace, de façon dépendante à la dose (300 et $500 \mathrm{mg} / \mathrm{kg})$, dans la prévention de l'œdème inflammatoire à la carraghénine. Toutefois, cet effet anti-inflammatoire est faible sur la phase initiale de l'œdème mais important dans la phase tardive $(6 \mathrm{~h})$.

L'œdème de la patte de rat induit par la carraghénine fait participer beaucoup de médiateurs qui induisent la réaction inflammatoire dans deux phases différentes (Garcia et al., 2004). Une phase initiale, qui se produit entre 0 et 2,5 heures après l'injection de l'agent phlogogène attribuée à l'action de médiateurs tels que l'histamine, la sérotonine et la bradykinine sur la perméabilité vasculaire (Maity et al., 1998). Une phase retard, qui est le résultat de la surproduction des prostaglandines dans les tissus, médiée par la cyclo-oxygénase (COX) (Gilligan et al., 1994) et qui peut continuer au-delà de 5 heures après injection de la carraghénine (Perez-Gurrero et al., 2001). Nos résultats et la nature biphasée de l'œdème de la patte induit par la carraghénine observés, permettent de proposer que l'activité significative dans la suppression de la deuxième phase de l'inflammation puisse être due aux médiateurs impliqués dans la phase tardive de l'œdème de la patte de rat induit par la carraghénine. Ceci suggère une probable implication des voies de la cyclo-oxygénase et de la lipoxygénase dans l'activité de l'extrait aqueux des feuilles de Elaeis guineensis.

En outre, nos résultats sur la caractérisation phytochimique de l'extrait aqueux des feuilles de Elaeis guineensis montre la présence de tanins hydrolysables et de tanins condensés, non hydrolysables (composés polyphénoliques), mais aussi des hétérosides flavonoïdes et des alcaloïdes. Ils vont dans le même sens que les travaux de (Sasidharan et al., 2010 ; Yin et al., 2013) qui ont en effet montré la présence de tanins, de flavonoïdes et d'alcaloïdes dans des extraits de feuilles de Elaeis guineensis. Ces composés, notamment les flavonoïdes possèderaient une action inhibitrice sur l'inflammation (Olaleye et al., 2004 ; Sani et al., 2014 ; Banerjee et al., 2014) qui passerait par l'inhibition de la formation des principaux médiateurs proinflammatoires du métabolisme de l'acide arachidonique via l'inhibition des cyclooxygénases et lipoxygénases (Kim et al., 2004; Yoon et Baek, 2005).

\section{Conclusion}

Cette présente étude a montré un effet anti-inflammatoire de l'extrait aqueux des feuilles de Elaeis guineensis sur le modèle de l'œdème inflammatoire à la carraghénine chez le rat. Cette activité serait plus importante à dose élevée et pourrait être lié à l'inhibition des cyclo-oxygénases et lipoxygénases dans la phase tardive de l'œdème inflammatoire à la carraghénine. Ces résultats justifient l'utilisation de Elaeis guineensis Jacq. en médecine traditionnelle africaine dans la prise en charge du processus inflammatoire.

Une purification de l'extrait aqueux pourrait permettre d'isoler le ou les principes actifs responsable(s) de ces effets. Et plus tard, la détermination des mécanismes moléculaires impliqués devrait suivre.

\section{CONFLIT D'INTÉRÊT}

Les auteurs déclarent qu'aucun conflit d'intérêt n'est lié à ce travail.

\section{CONTRIBUTIONS DES AUTEURS}

MS et MN ont initié ces travaux. MS a effectué les manipulations, exploité les résultats et rédigé le manuscrit. WD et AS ont supervisé l'extraction. EB a autorisé la réalisation de la partie chimique dans son laboratoire. FSB, MS, ANS, AMD et GYS ont contribué à l'évaluation de l'activité antiinflammatoire des extraits. 


\section{REMERCIEMENTS}

Les auteurs remercient les techniciens des laboratoires de Pharmacologie et Pharmacodynamie et de Pharmacognosie et Botanique de la Faculté de Médecine, de Pharmacie et d'Odontologie, Université Cheikh Anta Diop.

\section{REFERENCES}

Anyanji VU, Mohamed S, Zokti JA, Ado MA. 2013. Anti-inflammatory properties of oil palm leaf (Elaeis guineensis Jacq.) extract in aged rats. Int. J. Pharm. Pharm. Sci., 5(4): 134-136.

Banerjee S, Chanda A, Adhikari A, Das AK, Biswas S. 2014. Evaluation of phytochemical screening and anti inflammatory activity of leaves and stem of Mikania scandens (L.) wild. Annals of Medical and Health Sciences Research, 4(4): 532-536.

Coussens LM, Werb Z. 2002. Inflammation and cancer. Nature, 420(6917): 860-867.

Dandona P, Aljada A, Bandyopadhyay A. 2004. Inflammation: the link between insulin resistance, obesity and diabetes. Trends in Immunology, 25(1): 4-7.

Galanaud P. 2003. Inflammation et antiinflammatoires. La Revue du Praticien, 53(5): 476-477.

Garcia MD, Fernandez MA, Alvarez A, Saenz MT. 2004. Anti-nociceptive and antiinflammatory effect of the aqueous extract from leaves of Pimenta racemosa var. Ozua (Myrtaceae). J. Ethnopharmacol., 91(1): 69-73.

Gilligan JP, Lovato SJ, Erion MD, Jeng AY. 1994. Modulation of carrageenan-induced hind paw edema by substance $P$. Inflammation, 18(3): 285-292.

Gruca M, Van Andel TR, Balslev H. 2014. Ritual uses of palms in traditional medicine in sub-Saharan Africa: a review. J. Ethnobiol. Ethnomed., 10: 60.

Karin M, Lawrence T, Nizet V. 2006. Innate immunity gone awry: linking microbial infections to chronic inflammation and cancer. Cell, 124(4): 823-835.

Kim HP, Kun HS, Chang HW, Kang SS. 2004. Anti-inflammatory plant flavonoids and cellular action mechanisms. $J$. Pharmacol. Sci., 96(3): 229-245.

Maity TK, Mandal SC, Mukherjee PK. 1998. Studies on anti-inflammatory effect of Cassia tora leaf extract (fam leguminosae). Phytother. Res., 12(3): 221223.

Mohamed S. 2014. Oil Palm Leaf: A New Functional Food Ingredient for Health and Disease Prevention. J. Food Process. Technol., 5(2): 1-6.

Ndiaye M, Anselm E, Séne M, Diatta W, Dièye AM, Faye B, Schini-Kerth VB. 2010. Mechanisms underlying the endothelium-dependent vasodilatory effect of an aqueous extract of Elaeis guineensis jacq. (Arecaceae) in porcine coronary artery rings. Afr. J. Traditional, Complementary and Alternative Medicines, 7(2): 118-124.

Olaleye SB, Oke JM, Etu AK, Omotosho IO, Elegbe RA. 2004. Antioxidant and antiinflammatory properties of a flavonoid fraction from the leaves of Voacanga africana. Nigerian Journal of Physiological Sciences, 19(1): 69-76.

Owoyele BV, Owolabi GO. 2014. Traditional oil palm (Elaeis guineensis Jacq.) and its medicinal uses : A review. TANG, 4(3): 15-22.

Perez-Gurrero C, Herrera MD, Ortiz R, De Sotomayor MA, Fernandez MA. 2001. A pharmacological study of Cecropia obtusifolia Betrol aqueous extract. J. of Ethnopharmacol., 76(3): 279-284.

Pousset JL. 2004. Plantes medicinales d'Afrique - Comment les reconnaître et les utiliser. : Edisud, 287p. Aix-en-Provence.

Renfrey S, Downton C, Featherstone J. 2003. The painful reality. Nat. Rev. Drug Discov., 2(3): 175-176. 
Rosalina T, Mohamed S, Samaneh GF, Noordin MM, Goh YM. 2011. Polyphenol Rich Oil palm leaves extract reduce hyperglycaemia and lipid oxidation in STZ-rats. Int. Food Res. J., 18(1): 179188.

Sani YM, Musa AM, Pateh UU, Haruna AK, Yaro AH, Sani MB, Magaji MG. 2014. Phytochemical Screening and Preliminary Evaluation of Analgesic and AntiInflammatory Activities of the Methanol Root Extract of Cissus Polyantha. Bayero Journal of Pure and Applied Sciences, 7(1): 19-23.

Sasidharan S, Nilawatyi R, Xavier R, Latha LY, Amala R. 2010. Wound Healing Potential of Elaeis guineensis Jacq Leaves in an Infected Albino Rat Model. Molecules, 15(5): 3186-3199.

Winter CA, Risley FA, Nuss G. 1962. Carrageenin induced oedema in hand paw of the rat as assays anti-inflammatory drugs. Proc. Soc. Exp. Biol. Med., 111(62): 544-547.

Yin NS, Abdullah S, Phin CK. 2013. Phytochemical constituents from leaves of Elaeis guineensis and their antioxidant and antimicrobial activities. Int. J. Pharm. Pharmaceut. Sci., 5(4): 137-140.

Yoon JH, Baek SJ. 2005. Molecular targets of dietary polyphenols with antiinflammatory properties. Yonsei Med. J., 46(5): 585-596. 